\title{
EFECTOS HETERÓTICOS PARA EL CARÁCTER EXTRACTO ETÉREO EN LA SEMILLA DE ZAPALLO Cucurbita moschata DUCH.
}

\section{HETEROTIC EFFECTS FOR THE ETHER EXTRACT CHARACTER IN BUTTERNUT SQUASH Cucurbita moschata DUCH. SEED}

\author{
Magda Piedad Valdés ${ }^{1}$, Sanin Ortiz $^{2}$, Franco Alirio Vallejo ${ }^{3}$ \\ ${ }^{1}$ Ing. Agroindustrial, Ph.D. Universidad Nacional de Colombia - Sede Palmira, Colombia, e-mail: mpvaldesr@unal.edu.co; \\ ${ }^{2}$ Zootecnista, Ph.D. Universidad Nacional de Colombia - Sede Palmira, Colombia, e-mail: sortizg@unal.edu.co; ${ }^{3}$ Ing. Agróno- \\ mo Ph.D. Universidad Nacional de Colombia - Sede Palmira, Colombia, e-mail: favallejoc@unal.edu.co, Facultad de Ciencias \\ Agropecuarias, Universidad Nacional de Colombia. A.A. 237. Palmira, Valle del Cauca, Colombia.
}

Rev. U.D.C.A Act. \& Div. Cient. 17(2): 371-379, Julio-Diciembre, 2014

\section{RESUMEN}

El estudio tuvo como objetivo, evaluar los efectos heteróticos promedio, varietal y específico para el carácter producción de extracto etéreo en la semilla de Cucurbita moschata. Las evaluaciones, se realizaron en la Universidad Nacional de Colombia - Sede Palmira, municipio de Candelaria, Valle del Cauca, en dos semestres: segundo semestre de 2012 y primero de 2013. Se emplearon 21 genotipos, conformado por 6 progenitores y 15 cruzamientos directos y 5 plantas por repetición, bajo un diseño de bloques completos al azar, con 4 repeticiones. Se evaluaron los caracteres producción de extracto etéreo/planta (EEPP), porcentaje de extracto etéreo (EE), peso de semilla/fruto (PSPF), peso unidad de semilla (PUS) y número de frutos/planta (NFP), utilizando la metodología propuesta por Gardner y Eberhart (1966). La heterosis promedia fue el componente más importante, en razón a que explicó el $54 \%$ de la variación de la suma de cuadrados de la heterosis. El semestre donde mejor se expresó la heterosis promedia fue en el segundo de 2012, en donde se presentaron diferencias significativas para los caracteres EEPP, EE y PSPF, indicando que hay divergencia genética entre las progenies y los progenitores. Con base en los efectos de heterosis varietal, los mejores progenitores para el carácter EEPP fueron las introducciones 142 y 160. Con base en los efectos de heterosis específica, se destacaron con efecto significativo $\mathrm{P}<0,01$, en la especie $C$. moschata, para EEPP fueron los cruzamientos 308 x 142, 308 x 160 y 142 x 136 .

\section{SUMMARY}

The study aimed to evaluate the average heterotic effects, varietal and specific character to ether extract production in butternut squash seeds Cucurbita moschata. The field and laboratory research was conducted at Universidad Nacional de Colombia Palmira - municipality of Candelaria Valle del Cauca, in two semesters: second half of 2012 and first half of 2013. 21 genotypes namely, 6 parents and 15 direct crosses and 5 plants per replication under completely randomized design with 4 replications were used. Characters evaluated were oil production/plant (EEPP); percent of crude fat (EE) in seed; Seed Weight/fruit (PSPF); unit seed weight (PUS) and number of fruits / plant (NFP), using the method proposed by Gardner \& Eberhart (1966). The average heterosis was the most important component, as it explained $54 \%$ of the variation in the total sum of squares of heterosis. The term where heterosis was better expressed was the second half of 2012; significant differences $(P<0,05)$ for EEPP, EE and PSPF were displayed, indicating that there is genetic divergence between parents and offspring. Based on the effects of varietal heterosis, the best parents for EEPP introductions were 142 and 160. Based on the effects of specific heterosis, with significant effect, $P<0,01$, in $C$. mochata for EEPP, the crosses $308 \times 142,308 \times 160$ and $142 \times 136$ stand out.

Key words: Pumpkin squash, oilseed, heterosis, hybridization, dialelic crossing.

Palabras clave: Ahuyama, semilla oleaginosa, heterosis, hibridación, cruzamiento dialélico. 


\section{INTRODUCCIÓN}

El interés por la semilla de zapallo, como fuente de energía, ha ido creciendo, debido al alto contenido de extracto etéreo o grasa bruta (Ortiz et al. 2009).

Los principales estudios sobre semillas de Cucurbitas, se reportan en Grecia, donde encontraron que el aceite proveniente de Cucurbita pepo se destaca como fuente alternativa, para la producción de biodiesel (Schinas et al. 2009). Resultados experimentales mostraron que el contenido de aceite fue del $45 \%$ y el perfil de ácidos grasos del aceite mostró que está compuesto, principalmente, de ácidos linoleico, oleico, palmítico y esteárico.

En Austria, Eslovenia, Hungría y Serbia, la semilla proveniente de C. pepo y se utiliza en la elaboración de aceite, el cual, se produce a partir de dos variedades $C$. pepo var. styriaca Greb. y C. pepo var. oleífera (Idouraine et al. 1996). Durante el proceso, las semillas son tratadas con presión en frío, para extraer el aceite, dando como resultado alto rendimiento de aceite, que es utilizado en las comidas; la torta derivada del proceso, es empleada para la alimentación animal y como fertilizante (Pericin et al. 2009). Los componentes fenólicos de las semillas oleaginosas, así como en la pulpa y la cáscara (epicarpio) representan un potencial para la salud y para la industria (Peschel et al. 2006; Shahidi et al. 2006; Vuorela et al. 2003; Wang et al. 2007).

Estudios realizados en Colombia, reportaron que el aceite proveniente de la semilla de Cucurbita moschata Duch., sobresale por el contenido de ácidos grasos insaturados, $55,28 \%$ y una cantidad apreciable de ácido linoleico, 55,11\% (Ortiz et al. 2009). La torta proveniente de la semilla contiene $51,11 \%$ de proteína y $4604,60 \mathrm{kcal} / \mathrm{kg}$ de energía (Ortiz et al. 2009), similar a la reportada por Escobar et al. (2012), con 48,3\% de proteína, en la especie C. pepo.

La identificación de genotipos con efectos heteróticos para la producción de extracto etéreo no se ha explorado, por lo menos, en lo que respecta a la especie C. moschata (Valdés et al. 2013).

El concepto de heterosis no sólo se refiere al mayor incremento de los caracteres en la $F_{1}$, sino también puede ser la disminución de los mismos, dependiendo el carácter de interés, con relación a los progenitores (Hallauer \& Miranda, 1988; Ceballos, 1998; Vallejo et al. 2010). En zapallo C. moschata, la información sobre el comportamiento heterótico de los genotipos, se ha concentrado en el estudio del rendimiento de fruto fresco (Espitia et al. 2006) y en calidad y estabilidad genética del fruto para materia seca (Ortiz et al. 2009; Valdés et al. 2010); sin embargo, se desconoce si existen progenitores heteróticos para el carácter extracto etéreo en la semilla.
Gardner \& Eberhart (1966) propusieron un modelo que considera los efectos de cada progenitor y los efectos de la heterosis, discriminada en tres tipos: a) la heterosis media: es la diferencia entre el promedio de los cruzamientos y el de sus progenitores; b) la heterosis varietal: es la heterosis promedio con que contribuye un progenitor en los cruzamientos en que participa y, c) la heterosis específica de cada combinación particular de progenitores (Mendoza et al. 2010).

El presente artículo tiene por objetivo evaluar los efectos heteróticos promedio, varietal y específico, de seis progenitores, para producción de extracto etéreo, en semilla en $C$. moschata.

\section{MATERIALES Y MÉTODOS}

El trabajo de campo, se realizó entre el 2012 y 2013, en el Centro Experimental de la Universidad Nacional de Colombia, Sede Palmira - CEUNP, localizado en el municipio de Candelaria y en la Granja Mario González Aranda (MGA), ubicada en Palmira, Valle del Cauca (Colombia). La valoración macromolecular, se ejecutó en el Laboratorio de Semillas de la Universidad Nacional de Colombia - Sede Palmira.

Se evaluaron 21 genotipos, correspondientes a 6 progenitores seleccionados y 15 cruzamientos directos, utilizando un diseño de bloques completos al azar, con 4 repeticiones y 5 plantas por repetición. En cada repetición, se evaluaron las tres plantas centrales en madurez de cosecha (Valdés et al. 2010). Se utilizó un total de 840 plantas, 420 plantas por semestre, a una distancia de siembra entre y dentro de surco de $3 \mathrm{~m}$. Los progenitores fueron la introducción, 308 procedente de Colombia y las introducciones, 129, 142, 144, 136 y 160 , procedentes de Centro América.

Los cruzamientos dialélicos fueron evaluados en dos semestres consecutivos, segundo semestre de 2012 y primero de 2013.

En el segundo semestre de 2012, las condiciones ambientales del municipio de Candelaria, se caracterizaron por una precipitación pluvial promedio de $1100 \mathrm{~mm}, 76 \%$ de humedad relativa y temperatura entre $26^{\circ} \mathrm{C}$ a $34^{\circ} \mathrm{C}$. Por su parte, el primer semestre de 2013, se distinguió por ser atípico, con alta precipitación, que causó inundación en diferentes partes del cultivo, lo que ocasionó alta humedad relativa y temperaturas bajas en las horas de la mañana, altas al medio día y lluvia en las horas de tarde, con bajas temperaturas.

\section{Caracteres evaluados:}

Extracto etéreo/planta (EEPP): Es el producto del número de frutos por planta (NFP) por el peso de la semilla por fruto (PSF) por el porcentaje de extracto etéreo (EE) en la semilla, expresado en gramos $(\mathrm{g})$. 
Extracto etéreo (EE): Relación porcentual entre la muestra de semilla oleaginosa y el contenido de extracto etéreo de la misma, corregido por la materia seca de la muestra, a $105^{\circ} \mathrm{C}$ por 24 horas (AOAC, 1990).

Peso de semilla/fruto (PSPF): Peso promedio de la semilla, de tres frutos tomados al azar de cada introducción y acondicionada a $12 \%$ de humedad.

Peso unidad de la semilla (PUS): Peso promedio de 100 semillas tomadas al azar, en tres frutos de cada introducción y acondicionada a $12 \%$ de humedad.

Número frutos/planta (NFP): Promedio del número de frutos por planta, a partir de las cinco plantas de cada introducción.

\section{Análisis de la heterosis:}

La heterosis fue analizada utilizando la metodología propuesta por Gardner \& Eberhart (1966). Se empleó el modelo fijo, correspondiente a progenitores seleccionados y el método 2 , que considera a los progenitores y cruzamientos directos. El modelo empleado fue el siguiente:

$Y_{\mathrm{j} j^{\prime}}=\mu_{\mathrm{v}}+1 / 2\left(\mathrm{~V}_{\mathrm{j}}+\mathrm{V}_{\mathrm{j}}\right)+\theta_{\mathrm{j} \mathrm{j}^{\prime}}$

Donde:

$Y_{\mathrm{jj}}$ : valor promedio esperado de una variedad parental $(\mathrm{j}=\mathrm{j}$ ) o de un cruzamiento $\left(j \neq j{ }^{\prime}\right)$. Sí $j=j^{\prime}$, entonces $\theta=0$ y sí $j \neq$ $\mathrm{j}$, entonces $\theta=1$.

$\mu_{\mathrm{v}}$ : media de las $\mathrm{n}$ variedades parentales.

$\mathrm{V}_{\mathrm{j}}$ : efecto varietal, de la j-esíma variedad parental.

ij : efecto de heterosis que resulta cuando la variedad j se cruza con la variedad j'.

La heterosis total $\left(\mathrm{ij}^{\prime}\right)$ se descompuso en:

$\hat{h}_{i j^{\prime}}=\hat{h}+\left(h_{j}+h_{j}{ }^{\prime}\right)+S_{j j^{\prime}}$

Donde:

$\hat{h}$ : heterosis promedia

$h_{j}$ y $h_{j}:$ heterosis varietal de los padres j y j'

$\mathrm{S}_{\mathrm{j} j}$ : heterosis específica $\hat{\mathrm{h}}+\mathrm{h}_{\mathrm{j}}$

El análisis de varianza, se realizó según la metodología propuesta por Gardner \& Eberhart (1966 - Análisis II), incluyendo las fuentes de variación principales: repeticiones, genotipos, error y total. La fuente de variación genotipos, se descompuso en efectos de variedades $\left(V_{j}\right)$ y heterosis $\left(h_{j j}\right)$. A su vez, el efecto de heterosis se descompuso en heterosis promedia $(\hat{\mathrm{h}})$, heterosis varietal $\left(\mathrm{h}_{\mathrm{j}}\right)$ y heterosis específica $\left(\mathrm{S}_{\mathrm{ij}}\right)$. Para el procesamiento de la información, se utilizó el siguiente software: SAS-9.1 (versión Windows) SAS Institute Inc (2000) y Microsoft Office, Excel 2010.

\section{RESULTADOS Y DISCUSIÓN}

Los resultados de los progenitores y de los híbridos para cada una de las variables en estudio, se presenta en la tabla 1. Tanto los híbridos como los progenitores expresaron un comportamiento diferencial, según el semestre de siembra, pero sin un patrón específico, donde el semestre S1, correspondiente al segundo semestre de 2012, fue el más favorable para la mayoría de los caracteres evaluados.

Al comparar los promedios generales, se observó que los híbridos fueron superiores a los progenitores en los dos semestres, se destacó el segundo semestre de 2012-2, como el más favorable.

Para los caracteres EEPP, EE y PUS, el mejor progenitor fue la introducción 129, superior a la media general, en los dos semestres. En el primer semestre, para las variables PSPF y NFP, fueron los progenitores 142 y 144, respectivamente y, para el segundo semestre, los progenitores 129 y 308, respectivamente.

Los híbridos que sobresalieron para los caracteres EEPP, EE, PSPF, PUS y NFP, para el primer semestre (2012-2), fueron: 308x142, 308x160, 129x136, 129x160, 142x136. En el segundo semestre (2013-1), para los mismos caracteres, fueron: 129x142, 142x144, 129x160, 129x160 y 129x142, respectivamente.

En la tabla 2, se presenta el análisis de varianza para las variables en estudio, el cual, incluye como fuentes de variación: genotipos, variedades y heterosis, ésta última abarca la descomposición en heterosis promedio, heterosis varietal y heterosis específica.

Los efectos de los genotipos para las diferentes variables expresaron diferencias altamente significativas $(P<0,01)$ en los dos semestres, con excepción del carácter EE en la semilla que, en el primer semestre, no expresó diferencias entre genotipos, pero sí en el segundo semestre. Los efectos de variedades fueron altamente significativos, en los dos semestres, con excepción para los caracteres EEPP y EE, en el primer semestre. Los efectos de heterosis fueron altamente significativos, en los dos semestres, con excepción del carácter EEPP, que no tuvo efecto en el segundo semestre y el carácter $\mathrm{EE}$, que no tuvo efecto, en el primer semestre.

Lo anterior sugiere que existe diversidad genética entre los progenitores y los híbridos, es decir, entre los 21 genotipos, lo que indica que, por lo menos, un genotipo es diferente de todos los demás.

Los efectos de variedades indican que existe suficiente diversidad genética entre progenitores para los caracteres PSPF, 
Tabla 1. Promedios de los híbridos y progenitores para la producción de extracto etéreo por planta y sus componentes en $C$. moschata, para dos semestres (S1 y S2).

\begin{tabular}{|c|c|c|c|c|c|c|c|c|c|c|}
\hline \multirow{2}{*}{ HÍBRIDOS } & \multicolumn{2}{|c|}{$\begin{array}{l}\text { EEPP } \\
(\mathrm{g})\end{array}$} & \multicolumn{2}{|c|}{$\begin{array}{l}\mathrm{EE} \\
(\%)\end{array}$} & \multicolumn{2}{|c|}{$\begin{array}{l}\text { PSPF } \\
\text { (g) }\end{array}$} & \multicolumn{2}{|c|}{$\begin{array}{l}\text { PUS } \\
\text { (g) }\end{array}$} & \multicolumn{2}{|c|}{ NFP } \\
\hline & $\mathrm{S} 1$ & $\mathrm{~S} 2$ & $\mathrm{~S} 1$ & $\mathrm{~S} 2$ & $\mathrm{~S} 1$ & $\mathrm{~S} 2$ & $\mathrm{~S} 1$ & $\mathrm{~S} 2$ & $\mathrm{~S} 1$ & S2 \\
\hline $308 \times 129$ & 152,9 & 105,0 & 41,65 & 40,68 & 102,52 & 97,16 & 16,39 & 14,24 & 3,58 & 2,63 \\
\hline $308 X 142$ & 284,7 & 42,7 & 40,58 & 30,14 & 102,41 & 62,13 & 15,89 & 10,69 & 6,75 & 2,08 \\
\hline $129 \times 142$ & 201,7 & 170,1 & 43,90 & 35,52 & 99,34 & 95,04 & 15,16 & 15,44 & 4,75 & 5,00 \\
\hline $308 \times 144$ & 169,3 & 121,1 & 42,89 & 41,91 & 83,37 & 74,76 & 14,99 & 14,55 & 4,42 & 3,67 \\
\hline $129 \times 144$ & 219,5 & 119,3 & 44,79 & 42,67 & 111,36 & 105,71 & 15,80 & 15,71 & 4,42 & 2,67 \\
\hline $142 \times 144$ & 254,2 & 64,5 & 43,40 & 45,96 & 90,26 & 61,10 & 17,51 & 13,84 & 6,17 & 2,13 \\
\hline $308 \times 136$ & 202,2 & 76,5 & 44,13 & 42,77 & 99,54 & 85,62 & 15,22 & 15,33 & 4,54 & 2,08 \\
\hline $129 \times 136$ & 251,3 & 97,6 & 44,58 & 41,34 & 118,31 & 99,59 & 17,97 & 18,38 & 4,75 & 2,42 \\
\hline $142 \times 136$ & 270,3 & 89,2 & 42,43 & 42,22 & 77,77 & 65,17 & 15,47 & 13,82 & 8,08 & 3,33 \\
\hline $144 X 136$ & 167,7 & 71,0 & 41,92 & 35,63 & 90,96 & 68,30 & 14,28 & 13,98 & 4,58 & 2,75 \\
\hline $308 \times 160$ & 252,9 & 77,3 & 46,67 & 38,82 & 79,88 & 65,03 & 13,92 & 14,86 & 6,83 & 3,00 \\
\hline $129 \times 160$ & 247,8 & 158,8 & 44,18 & 42,47 & 106,89 & 107,23 & 19,42 & 18,62 & 5,25 & 3,42 \\
\hline $142 \times 160$ & 229,3 & 120,8 & 46,06 & 40,41 & 84,91 & 72,30 & 15,54 & 16,70 & 6,08 & 4,00 \\
\hline $144 X 160$ & 210,6 & 145,2 & 41,29 & 43,32 & 96,57 & 95,67 & 13,36 & 14,70 & 4,92 & 3,50 \\
\hline $136 X 160$ & 190,0 & 117,8 & 41,61 & 41,26 & 85,03 & 86,49 & 21,27 & 18,33 & 5,50 & 3,25 \\
\hline $\begin{array}{l}\text { Promedio de } \\
\text { híbridos }\end{array}$ & 220,3 & 105,1 & 43,34 & 40,34 & 95,28 & 82,75 & 16,14 & 15,28 & 5,38 & 3,06 \\
\hline \multicolumn{11}{|l|}{$\begin{array}{l}\text { PROGE- } \\
\text { NITORES }\end{array}$} \\
\hline 308 & 106,0 & 85,3 & 38,04 & 43,95 & 52,58 & 43,23 & 12,46 & 14,43 & 5,42 & 4,92 \\
\hline 129 & 177,0 & 183,7 & 44,30 & 44,68 & 87,58 & 95,09 & 19,82 & 19,27 & 4,46 & 4,38 \\
\hline 142 & 173,3 & 40,3 & 41,87 & 37,50 & 100,07 & 64,29 & 13,33 & 10,76 & 4,25 & 1,63 \\
\hline 144 & 153,0 & 84,7 & 40,49 & 40,48 & 49,89 & 57,80 & 10,44 & 10,50 & 7,58 & 3,50 \\
\hline 136 & 133,2 & 53,4 & 37,61 & 30,56 & 67,03 & 68,18 & 18,14 & 15,15 & 4,33 & 2,29 \\
\hline 160 & 160,4 & 71,9 & 40,42 & 43,81 & 75,48 & 58,51 & 14,56 & 12,95 & 5,42 & 2,71 \\
\hline $\begin{array}{l}\text { Promedio } \\
\text { Progenitores }\end{array}$ & 150,5 & 86,5 & 40,45 & 40,16 & 72,11 & 64,52 & 14,79 & 13,84 & 5,24 & 3,24 \\
\hline
\end{tabular}

PUS y NFP, pero para los caracteres EEPP y porcentaje de EE, se percibe poca diversidad genética entre progenitores, teniendo en cuenta la no significancia en el primer semestre, para EEPP y porcentaje de EE.

Los efectos de heterosis indican que existe suficiente variabilidad para PSPF, PUS y NFP, pero poca diversidad para EEPP y porcentaje de EE, teniendo en cuenta la no significancia de EEPP, en el segundo semestre y porcentaje de EE, en el primer semestre.

Los efectos de variedades fueron no significativos $(\mathrm{P}<0.05)$, en el primer semestre y los efectos de heterosis fueron sig- nificativos $(P<0.05)$; caso contrario ocurrió con $E E$, lo que indica que hay un marcado efecto de semestre para las dos variables de mayor interés, en esta investigación. Por lo tanto, sería conveniente seleccionar genotipos para regiones con condiciones climáticas determinadas, según lo previsto por Ortiz et al. (2009).

La descomposición de los efectos de la heterosis en heterosis promedia, varietal y específica reveló que, la heterosis promedia de estos caracteres, indicó que los híbridos son superiores a los progenitores, por tanto, existe divergencia genética entre los progenitores, facilitando el proceso de mejoramiento genético para los mismos. La heterosis pro- 


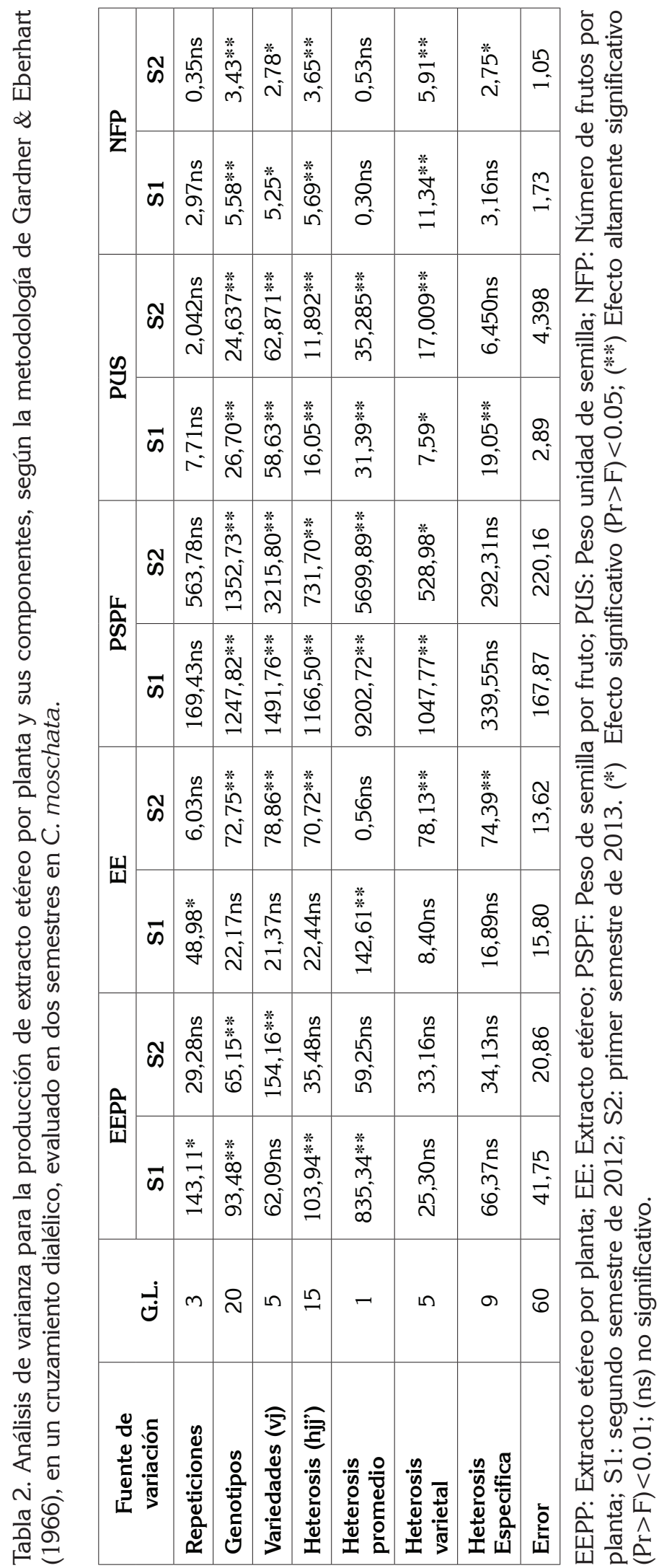


media fue el componente más importante, en razón a que explicó el 54\% de la variación de la heterosis.

La complementariedad genética reducida entre los progenitores para los caracteres EEPP y porcentaje de EE, sobretodo en el primer semestre, no provocó efecto diferencial para heterosis varietal. Para NFP, a pesar de la reducida variabilidad entre los progenitores, fue suficiente para provocar efecto diferencial para heterosis varietal.

La ausencia de significancia para heterosis específica para los caracteres EEPP, EE, PSPF y NFP indicó que no hubo efecto favorable en la complementariedad genética de los cruzamientos en que participaron o porque no se presentó el efecto de dominancia, posiblemente, porque las frecuencias génicas de algunos progenitores no difieren significativamente o porque hubo dominancia positiva para algunos locus y negativa para otros, lo cual, termina anulando o impidiendo la expresión de la heterosis específica, por lo tanto, es la dominancia direccional la que permite detectar la heterosis. Como las progenies de cada uno de los progenitores son similares, se puede seleccionar dentro de la superioridad hibrida un hibrido con heterosis.

\section{Estimación de efectos de heterosis:}

Efecto de heterosis promedia: En la tabla 3, se presenta el efecto de la heterosis promedia, para los caracteres producción de extracto etéreo por planta y sus componentes. La heterosis promedia es la diferencia entre el promedio de los híbridos y el promedio de los progenitores. El semestre donde mejor se expresó la heterosis promedia fue en el primero, en donde se presentaron diferencias significativas, para los caracteres EEPP, EE y PSPF, asimismo, para PSPF, pero en el segundo semestre, indicando que existe divergencia genética entre las progenies y los progenitores. La ausencia de significancia en los demás caracteres indicó que, en conjunto, esas variedades usadas no son favorables para explorar el efecto de heterosis en los híbridos.

Efecto de heterosis varietal: La heterosis varietal es la diferencia entre el promedio de todos los híbridos derivados de un progenitor y el promedio de todos los híbridos. En la tabla 4 , se presentan los efectos varietales para todas las variables en estudio.

Tabla 3. Efecto de heterosis promedia para el carácter producción de extracto etéreo por planta y sus componentes, en dos semestres (S1: 2012-2, S2: 2013-1), para la especie C. moschata.

\begin{tabular}{|l|c|c|}
\hline \multicolumn{1}{|c|}{ CARÁCTER } & S1 & S2 \\
\hline EEPP & $6,98^{*}$ & 1,86 \\
\hline EE & $2,88^{*}$ & 0,18 \\
\hline PSPF & $23,17^{*}$ & $18,23 *$ \\
\hline PUS & 1,35 & 1,43 \\
\hline NFP & 0,13 & $-0,17$ \\
\hline
\end{tabular}

(*) Efecto significativo $(\operatorname{Pr}>\mathrm{F})<0.05$.

Tabla 4. Efectos de heterosis varietal para el carácter producción de extracto etéreo por planta y sus componentes, en dos semestres (S1: 2012-2, S2: 2013-1), para la especie C. moschata.

\begin{tabular}{|c|c|c|c|c|c|c|c|c|c|c|}
\hline \multirow{3}{*}{$\begin{array}{l}\text { VARIE- } \\
\text { DADES }\end{array}$} & \multicolumn{10}{|c|}{ Efecto de heterosis varietal } \\
\hline & \multicolumn{2}{|c|}{ EEPP } & \multicolumn{2}{|c|}{$\mathrm{EE}$} & \multicolumn{2}{|c|}{ PSPF } & \multicolumn{2}{|c|}{ PUS } & \multicolumn{2}{|c|}{ NFP } \\
\hline & S1 & S2 & S1 & S2 & S1 & S2 & S1 & S2 & S1 & S2 \\
\hline 308 & 1,24 & $-2,52$ & 1,01 & $-3,74$ & 7,60 & 3,38 & 0,09 & $-1,97$ & $-0,27$ & $-1,30$ \\
\hline 129 & $-2,03$ & $-1,73$ & $-1,32$ & $-2,02$ & 7,77 & 7,45 & $-1,51$ & $-1,22$ & $-0,64$ & $-0,36$ \\
\hline 142 & 2,33 & 1,35 & $-0,79$ & $-0,53$ & $-19,40$ & $-14,39$ & 0,44 & 0,06 & 1,74 & 1,11 \\
\hline 144 & $-2,13$ & $-0,02$ & $-0,62$ & 1,79 & 10,14 & 1,30 & 0,98 & 0,77 & $-1,76$ & $-0,28$ \\
\hline 136 & 0,37 & $-0,18$ & 0,92 & 5,18 & 1,35 & $-3,98$ & $-0,81$ & 0,21 & 0,60 & 0,10 \\
\hline 160 & 0,23 & 3,09 & 0,80 & $-0,68$ & $-7,46$ & 6,24 & 0,81 & 2,15 & 0,34 & 0,73 \\
\hline
\end{tabular}


Los efectos varietales variaron entre semestres. Para el primer semestre, se presentó:

- Las variedades 308 y 142 presentaron efectos positivos favorables para el carácter EEPP y las variedades 308 , 136 y 160 para porcentaje de EE, lo que indica que estos progenitores permiten obtener cruzamientos con mayor producción de EEPP y mayor contenido de extracto etéreo en la semilla, respectivamente.

- Las variedades 308, 129, 144 y 136 presentaron valores positivos para el carácter PSPF y las variedades 144 y 160 presentaron valores positivos, para el carácter PUS, indicando que estas variedades tienen mayor potencial para obtener cruzamientos con mayor PSPF y PUS, respectivamente.

- Las variedades 142,136 y 160 presentaron valores positivos para el NFP, lo que indicó que estas variedades son las de mayor potencial para ser empleadas en cruzamientos, para incrementar el carácter NFP.

Para el segundo semestre, las variedades que tuvieron efectos positivos fueron:

- Para el carácter EEPP: 142 y 160

- Para el carácter EE: 144 y 136

- Para el carácter PSPF: 308, 129, 144 y 160
- Para el carácter PUS: 144 y 160

- Para el carácter NFP: 142 y 160

Efecto de heterosis específica: El efecto de heterosis especifica expresa la diferencia entre cada cruzamiento individual con respecto a lo esperado, con base en la heterosis promedia y la heterosis varietal de sus progenitores (Ceballos, 1998).

En la tabla 5, se presentan los efectos de heterosis específica, los cuales, variaron entre semestres. Para el carácter EEPP, los híbridos 308x142, 308x160 y 142x136, presentaron diferencias positivas y significativas, para el primer semestre, indicando que esos híbridos fueron superiores al promedio de sus progenitores y a la media general; en el segundo semestre, a pesar de que se obtuvieron valores positivos, no fueron significativos para este carácter.

El hibrido 308x160, se destacó para el carácter EE, en el primer semestre, por presentar valores positivos y significativos $\mathrm{y}$, en el segundo semestre, los híbridos que se destacaron fueron $142 \times 144$ y $142 \times 136$, lo que sugiere que estos híbridos son los más indicados para explorar el efecto heterótico.

Tabla 5. Efectos de heterosis específica para el carácter producción de extracto etereo por planta y sus componentes, en dos semestres (S1:2012-2 y S2:2013-1), para la especie C. moschata.

\begin{tabular}{|c|c|c|c|c|c|c|c|c|c|c|c|c|}
\hline \multirow{3}{*}{$\begin{array}{l}\text { HÍBRI- } \\
\text { DOS }\end{array}$} & \multicolumn{12}{|c|}{ Efecto de heterosis específica } \\
\hline & \multicolumn{2}{|c|}{ EEPP } & \multicolumn{2}{|c|}{$\mathrm{EE}$} & \multicolumn{4}{|c|}{ PSPF } & \multicolumn{2}{|c|}{ PUS } & \multicolumn{2}{|c|}{ NFP } \\
\hline & S1 & S2 & S1 & S2 & S1 & & S2 & & S1 & $\mathrm{S} 2$ & S1 & S2 \\
\hline $308 \times 129$ & $-5,05 \mid n s$ & $-0,57 \mathrm{~ns}$ & $-2,10$ ns & $1,94 \mathrm{~ns}$ & $-6,11=$ & $*$ & $-1,07$ & $*$ & 0,32 ns & $-0,86$ ns & $-0,57$ ns & $-0,18$ ns \\
\hline $308 \times 142$ & $3,96 *$ & $-2,71 n s$ & \begin{tabular}{|l|l}
$-2,48$ ns \\
\end{tabular} & $-6,49$ ns & & * & 1,15 & ns & \begin{tabular}{l|l}
$1,11 \mathrm{~ns}$ \\
\end{tabular} & $-1,43$ ns & 0,32 ns & \begin{tabular}{|l|l}
$-0,82$ & $n s$ \\
\end{tabular} \\
\hline $308 \times 144$ & $-2,11 \mathrm{~ns}$ & $4,29 \mathrm{~ns}$ & $0,34 \mathrm{~ns}$ & $1,47 \mathrm{~ns}$ & $-8,78$ & * & 1,33 & ns & $1,12 *$ & $1,86 \mathrm{~ns}$ & $-0,18$ ns & \begin{tabular}{|l|l|l}
$1,22 \mathrm{~ns}$ \\
\end{tabular} \\
\hline $308 \times 136$ & $-0,32$ ns & $1,55 \mathrm{~ns}$ & $1,49 \mathrm{~ns}$ & $3,90 \mathrm{~ns}$ & 7,62 & * & 12,28 & $*$ & $-0,71$ ns & 0,87 ns & $-0,79$ ns & $-0,15 \mathrm{~ns}$ \\
\hline $308 \times 160$ & $3,52 *$ & $-2,57 \mathrm{~ns}$ & $2,75 *$ & \begin{tabular}{|l|l|}
$-0,82$ & ns \\
\end{tabular} & $-7,46 \mathrm{r}$ & ns & $-13,69$ & ns & $-1,84 \mathrm{~ns}$ & $-0,44$ ns & 1,22 ns & $-0,06$ ns \\
\hline $129 \times 142$ & $-4,62$ ns & $4,33 \mathrm{~ns}$ & $0,04 \mathrm{~ns}$ & $-3,20$ ns & $-6,02 \mathrm{r}$ & ns & 4,06 & ns & $-1,70$ ns & 0,14 ns & $-0,83$ ns & $1,43 *$ \\
\hline $129 \times 144$ & 2,63 ns & $-1,60$ ns & $1,45 \mathrm{~ns}$ & $0,14 \mathrm{~ns}$ & 1,54 & $*$ & 2,27 & $*$ & $-0,15$ ns & $-0,16$ ns & 0,67 ns & $-0,45$ ns \\
\hline $129 \times 136$ & 4,31 ns & $-2,04$ ns & $1,15 \mathrm{~ns}$ & 0,38 ns & 8,72 & $*$ & $-3,76$ & ns & $-0,05$ ns & 0,74 ns & $0,26 \mathrm{~ns}$ & \begin{tabular}{|l|l|}
$-0,48$ ns \\
\end{tabular} \\
\hline $129 \times 160$ & 2,73 ns & \begin{tabular}{|l|l}
$-0,12$ ns \\
\end{tabular} & \begin{tabular}{|c|c|}
$-0,54$ \\
ns
\end{tabular} & $0,74 \mathrm{~ns}$ & $1,88 \mathrm{r}$ & ns & $-1,50$ & ns & 1,58 ns & 0,14 ns & \begin{tabular}{l|l}
$0,48 \mathrm{~ns}$ \\
\end{tabular} & \begin{tabular}{|l|l|}
$-0,31$ ns \\
\end{tabular} \\
\hline $142 \times 144$ & 1,92 ns & $-2,99$ ns & 0,74 ns & $5,54 *$ & $1,37 \mathrm{r}$ & & $-5,09$ & ns & $2,85 *$ & 0,94 ns & 0,15 ns & $-1,10$ ns \\
\hline $142 \times 136$ & $2,03 *$ & $1,20 \mathrm{~ns}$ & $-0,33$ ns & $3,37 *$ & & ns & $-0,93$ & ns & $-1,26$ ns & $-0,84$ ns & $1,32 *$ & $0,33 \mathrm{~ns}$ \\
\hline $142 \times 160$ & $-3,30 \mathrm{~ns}$ & 0,17 ns & 2,02 ns & $0,79 \mathrm{~ns}$ & $0,83 \mathrm{r}$ & ns & 0,81 & ns & $-1,01$ ns & $1,19 *$ & $-0,96$ ns & $0,16 *$ \\
\hline $144 \times 136$ & $-2,76$ ns & \begin{tabular}{|l|l|}
$-1,46 \mathrm{~ns}$ \\
\end{tabular} & \begin{tabular}{|l|l} 
& $-0,31$ \\
ns
\end{tabular} & \begin{tabular}{|l|l|}
$-7,04$ & $\mathrm{~ns}$ \\
\end{tabular} & $-2,16:$ & & $-10,24$ & ns & $-1,54$ ns & $-1,26$ ns & $-0,34$ ns & $0,21 \mathrm{~ns}$ \\
\hline $144 \times 160$ & $0,31 \mathrm{~ns}$ & $1,76 \mathrm{~ns}$ & $-2,23$ ns & $-0,11$ ns & $8,04:$ & & 11,73 & $*$ & $-2,28$ ns & $-1,37$ ns & $-0,29$ ns & 0,12 ns \\
\hline $136 \times 160$ & $-3,26$ ns & $0,75 \mathrm{~ns}$ & $-2,00$ ns & $-0,60$ ns & $-3,28 \mathrm{r}$ & & 2,65 & & $3,56 *$ & $0,48 *$ & $-0,45$ ns & $0,09 \mathrm{~ns}$ \\
\hline
\end{tabular}


Los efectos de heterosis específica, para el carácter PSPF, presentaron valores positivos y significativos en los híbridos 308x142, 308x136, 129x144, 129x136 y 144x160, para el primer semestre, indicando que estos híbridos son propicios para incrementar el PSPF. Los valores negativos y significativos de los híbridos 308x129, 308x144 y 144x136, sugieren que sus promedios fueron inferiores al promedio esperado, con base en la media general, lo que permite deducir que no existe complementación genética entre sus progenitores, a pesar de que éstos tienen origen geográfico diferente.

Hubo diferencias significativas y positivas para el carácter PUS, en el primer semestre, para los híbridos 308x144, $142 x 144$ y 136x160 y, para el segundo semestre, los mejores híbridos fueron el 142x160 y 136x160, lo que indica que se puede explorar la heterosis en estos híbridos, para aumentar el PUS.

Hubo diferencia significativa y positiva para el carácter NFP, en el primer semestre, para el hibrido $142 \times 136$ y, en el segundo, en los híbridos 129×142 y 142×160, lo que indicó que es posible incrementar el NFP, si se explora en estos híbridos.

Bajo las condiciones de estudio, las variables EEPP, porcentaje de EE, PSPF y PUS, presentaron efectos de heterosis promedia altamente significativos, en el segundo semestre de 2012, indicando que hubo divergencia genética entre progenitores.

Los mejores progenitores para los componentes de EEPP fueron las introducciones 142 y 160, teniendo en cuenta los efectos de heterosis varietal.

Los progenitores 308 y 142 presentaron efectos de heterosis varietal positivos, para el carácter EEPP, en el primer semestre y los progenitores 142 y 160 , en el segundo. Para el porcentaje de EE, en el primer semestre, los progenitores sobresalientes fueron 308, 136 y 160 y, en el segundo, los progenitores 136 y 144. Para el PSPF, en el primer semestre, los progenitores sobresalientes fueron 308, 129 y 144 y, en el segundo, los progenitores 308, 129 y 160.

Para el carácter EEPP, los híbridos 308x142, 308x160 y $142 \times 136$, fueron superiores al promedio de sus progenitores, para el primer semestre; en el segundo, a pesar que se obtuvieron valores positivos, no fueron significativos. El hibrido 308x160, se destacó para el carácter EE, en el primer semestre $y$, en el segundo, los híbridos que se destacaron fueron $142 \times 144$ y 142x136.

De este estudio, se concluyó que, basado en la heterosis promedio, se evidenció que existe diversidad genética entre los progenitores y sus progenies en la especie C. moschata, para los componentes asociados a la producción de extracto etéreo por planta EEPP.

Con base en los promedios y los efectos de heterosis varietal, los mejores progenitores para los componentes de EEPP son las introducciones 142 y 160 y con base en los promedios y los efectos de heterosis específica, los mejores híbridos para los componentes de EEPP son los cruzamientos $308 \times 142$, 308 x 160 y 142 x 136 .

Agradecimientos: Los autores agradecen al programa Mejoramiento genético, agronomía y producción de semillas de hortalizas de la Universidad Nacional de Colombia - Sede Palmira. Conflicto de intereses: El manuscrito fue preparado y revisado con la participación de todos los autores, quienes declaramos que no existe conflicto de intereses, que ponga en riesgo la validez de los resultados presentados.

\section{BIBLIOGRAFÍA}

1. AOAC. 1990. Official Methods of Analysis of the Association of Official analytical chemists. $15 \mathrm{Ed}$. Arlington, Virginia, USA. Método número 920.39.

2. CEBALLOS, L.H. 1998. Genética Cuantitativa y Fitomejoramiento. Universidad Nacional de Colombia Sede Palmira. 524p.

3. ESCOBAR, G.D.V.; CURUTCHET, A.; ZIRBESEGGER, H.; MÁRQUEZ, R.R. 2012. Estudio de la composición fisicoquímica de harina de semillas de zapallo como ingrediente alimentario. Rev. Lab. Tecnológico Uruguay. 7:25-30.

4. ESPITIA, C.M.M.; VALLEJO, C.F.A.; ARAMENDIZ, H. 2006. Evaluación agronómica de siete híbridos experimentales F1 de zapallo (Cucurbita moschata Duch. EX POIR). Temas Agrarios. 11(1):32-42.

5. GARDNER, C.O.; EBERHART, S.A. 1966. Analysis and interpretation of the variety cross diallel and related populations. Biometrics: 22:439-452.

6. HALLAUER, A.R.; MIRANDA, J.B. 1988. Quantitative Genetics in Maize-Breeding. lowa State University Press, Ames (10). 468p.

7. IDOURAINE, A.; KOHLHEPP, E.A.; WEBER, C.W.; WARID, W.A.; MARTINEZ-TELLEZ, J.J. 1996. Nutrient constituents from eight lines of naked seed squash (Cucurbita pepo L.). J. Agr. Food Chem. 44:721-724. 
8. MENDOZA, DE JESÚS V.; SAHAGÚN-CASTELLANOS, J.; RODRÍGUEZ-PÉREZ, J.E.; LEGARIA-SOLANO, J.P.; PEÑA-LOMELÍ, A.; PÉREZ-GRAJALES, M. 2010. Heterosis intervarietal en jitomate de crecimiento indeterminado tipo saladete. Rev. Chapingo Ser. Hortic, Chapingo, 16(1), Disponible desde Internet en http://www.scielo.org.mx/scielo.php?script=sci arttext\&pid=S1027-152X2010000100008\&lng $=\mathrm{es}^{-}$ $\& n r m=$ iso (con acceso 09/01/2014).

9. ORTIZ, G.S.; PASOS, L.S.C.; RIVAS, A.X.C.; VALDÉS, R.M.P.; VALLEJO, C.F.A. 2009. Extracción y caracterización de aceite de semillas de zapallo. Acta Agronómica 58(3):145-151.

10. PERICIN, D.; KRIMER, V.; TRIVIC, S.; RADULOVIC, L. 2009. The distribution of phenolic acids in pumpkin's hull-less seed, skin, oil cake meal, dehulled kernel and hull. J. Food Chem. 113(2):450-456.

11. PESCHEL, W.; SANCHEZ-RABANEDA, F.; DIEKMANN, W.; PLESCHER, A.; GARTZIA, I.; JIMENEZ, D. 2006. An industrial approach in the search of natural antioxidants from vegetable and fruit wastes. Food Chemistry. 97(1):137-150.

12. SAS Institute. 2000. SAS campus drive. Cary, NC.

13. SCHINAS, P.; KARAVALAKIS, G.; DAVARIS, C.; ANASTOPOULOS, G.; KARONIS, D.; ZANNIKOS, F.; STOURNAS, S.; LOIS, E. 2009. Pumpkin (Cucurbita pepo L.) seed oil as an alternative feedstock for the production of biodiesel in Greece. Biomass Bioenerg. 33(1):44-49.
14. SHAHIDI, F.; LIYANA-PATHIRANA, C.M.; WALL, D.S. 2006. Antioxidant activity of white and black sesame seeds and their hull fractions. Food Chemistry. 99(3):478-483.

15. VALDÉS, R.M.P.; ORTIZ, G.S.; BAENA, G.D.; VALLEJO, C.F.A. 2010. Evaluación de poblaciones de zapallo Cucurbita moschata Duch. por caracteres de importancia agroindustrial. Acta Agr. 59(1):91-96.

16. VALDÉS, R.M.P.; ORTIZ, G.S.; BAENA, G.D.; VALLEJO, C.F.A. 2013. Evaluación de siete poblaciones de zapallo para caracteres de importancia agroindustrial. En: Ortiz, G.S. (ed.) Zapallo para consumo en fresco y fines agroindustriales: investigación y desarrollo. Cali. Feriva. 250p.

17. VALLEJO, C.F.A.; ESPITIA, C.M.; ESTRADA, E.I.; RAMÍREZ H. 2010. Genética Vegetal. Universidad Nacional de Colombia Sede Palmira. Editorial Feriva S.A. Colombia. 383p.

18. VUORELA, S.; MEYER, A.S.; HEINONEN, M. 2003. Quantitative analysis of the main phenolics in rapeseed meal and oils processed differently using enzymatichydrolysis and HPLC. European Food Res. Technol. 217(6):517-523.

19. WANG, J.; YAUN, X.; JIN, Z.; TIAN, Y.; SONG, H. 2007. Free radical and reactive oxygen species scavenging activities of peanut skins extracts. Food Chemistry. 104(1):242-250.

Recibido: Abril 6 de 2014

Aceptado: Agosto 13 de 2014

Como citar:

Valdés, M.P.; Ortiz, S.; Vallejo, F.A. 2014. Efectos heteroticos para el carácter extracto etéreo en la semilla de zapallo Cucurbita moschata Duch. Rev. U.D.C.A Act. \& Div. Cient. 17(2): 371-379. 